\title{
Covariant GAUGES FOR CONSTRAINED SYSTEMS
}

\author{
S. A. Gogilidze, ${ }^{*}$ A. M. Khvedelidze, ${ }^{\dagger}$ V. N. Pervushin
}

The method of constructing of extended phase space for singular theories which permits the consideration of covariant gauges without the introducing of a ghost fields, is proposed. The extension of the phase space is carried out by the identification of the initial theory with an equivalent theory with higher derivatives and applying to it the Ostrogradsky method of Hamiltonian description .

JINR Preprint E2-95-132

\footnotetext{
${ }^{1 *}$ Permanent address: Tbilisi State University, 380086, Tbilisi, Georgia.

${ }^{2 \dagger}$ Permanent address: Tbilisi Mathematical Institute, 380093, Tbilisi, Georgia, Electronic address: khved@theor.jinrc.dubna.su
} 


\section{INTRODUCTION}

The crucial point for understanding the physical content of gauge theories consist in the identification of field degrees of freedom with observable quantities. The direct way to achieve this is to handle only with physical degrees of freedom. To realize this program at the classical level one can pass to the theory without redundant variables. This elimination of redundant variables - the reduction of initial theory, can be carried out in the configuration or phase space. Correspondingly, we have the Lagrangian or Hamiltonian form of reduction. In the former we restrict the configuration space to the consideration of the gauge fields obeying the Lagrangian constraint equations. As a result, nonphysical variables disappear from gauge invariant quantities and the remaining physical variables describe a usual unconstrained system admitting the Hamiltonian description. For realization of the Lagrangian reduction it is necessary to deal with the explicit solution of constraints. But it is not such as a straightforward procedure. In the first place, in general it involves a solving of complicated differential equations. Secondly, apart ifrom these practical difficulties there is a desire to maintain the manifest covariance of the initial gauge theory which is immediately broken after resolution of the Lagrangian constraints. To avoid these disadvantages connected with the resolution of constraints in the Lagrangian reduction scheme, one commonly uses the Dirac description for generalized Hamiltonian systems [1] - [3], based on the notion of weak equations. In this case, the Hamiltonian reduction consists in the elimination of these week equations. The elimination of weak equations is achieved by gauge - fixing procedure: introduction into the theory of some new "gauge constraints" and replacement of the Poisson bracket by the Dirac one. According to the Dirac method, the gauge condition is an arbitrary function of coordinates and momenta alone. This gauge type of gauge conditions has been called the unitary gauges [3]. However, the class of unitary gauges is not sufficiently large. The so - called covariant gauges (e.g. the Lorentz gauge $\partial_{\mu} A^{\mu}=0$ ) containing the velocities, which are alien to the Hamiltonian description, turned out it. So the manifest covariance is again unattainable. There are known methods how to overcome this difficulty. It has been shown [4] that one can always consider the gauge with explicit time dependence which is equivalent to the velocity depending gauge

and, thus reduce the problem to the case of unitary gauges with explicit time dependence. Another approach has been established in ref. [5] where with the aim to include this class of 
gauges in the consideration the phase space of the initial system is extended by introducing the auxiliary fermionic fields (ghost fields).

In the present note we suggest an alternative approach to consideration of covariant gauges. In contrast with the approach [5], the phase space for degenerate theory is constructed without introducing ghost fields, by the identification of the initial theory with the equivalent theory with higher derivatives and applying to it the Ostrogradsky method of Hamiltonian description [3], [6]. We will construct the extended phase space ( without ghosts ) and demonstrate that the gauge fixing condition, which contains a velocity and thus is nonunitary in ordinary phase space, represents a usual unitary gauge in the obtained extended phase space.

The remainder of this article is organized as follows. In the next section, we shall briefly describe the gauge fixing method according to Dirac's scheme. Section 3 is devoted to the Ostrogradsky method of construction of the phase space for systems with higher derivatives. (the Legendre transformation generalization) In section 4 we describe our scheme. And in the last section we consider the application of this scheme to the case of relativistic particle.

\section{DIRAC'S METHOD OF REDUCTION : UNITARY GAUGE FIXING}

Let us recall briefly the main points of construction of the Dirac Hamiltonian description for degenerate systems. Suppose, that the system with finite number of degrees of freedom has the following first class constraints $(\alpha=1, \ldots, m)$

$$
\phi_{\alpha}(p, q)=0, \quad\left\{\phi_{\alpha}(p, q), \phi_{\beta}(p, q)\right\}=f_{\alpha \beta \gamma}(p, q) \phi_{\gamma}(p, q)
$$

The generalized Hamiltonian dynamics is described by the extended Hamiltonian

$$
H_{E}=H_{C}+u_{\alpha}(t) \phi_{\alpha}(p, q)
$$

where the $H_{C}$ is canonical Hamiltonian and $u_{\alpha}$ are the Lagrange multipliers. According to the Dirac gauge fixing prescription one must introduce the new "gauge " constraints

$$
\chi_{\alpha}(p, q)=0
$$

with the requirement

$$
\operatorname{det}\left\{\chi_{\alpha}, \phi_{\beta}\right\} \neq 0
$$

From the maintenance of these auxiliary conditions $(\sqrt{2.3})$ in time it follows the set of equations

$$
0 \equiv \dot{\chi}_{\alpha}=\left\{\chi_{\alpha}, H_{C}\right\}+\sum_{\beta}\left\{\chi_{\alpha}, \phi_{\beta}\right\} u_{\beta}=0
$$


which allows determining the unknown Lagrange multipliers. Formally, the solution can be written as

$$
u_{\beta}=-\sum\left\{H_{C}, \chi_{\alpha}\right\} C_{\alpha \beta}^{-1} \phi_{\beta},
$$

where $C^{-1}$ is the inverse matrix of

$$
C_{\alpha \beta}=\left\{\chi_{\alpha}, \phi_{\beta}\right\}, \quad C_{\alpha \beta} C_{\beta \gamma}^{-1}=\delta_{\alpha \gamma}
$$

The Dirac elegant observation consists in that instead of the determination in such a way the unknown function $u_{\alpha}(t)$, one can change the Poisson brackets to the new - Dirac one

$$
\{F, G\}_{D}=\{F, G\}-\left\{F \chi_{\alpha}\right\} C_{\alpha \beta}^{-1}\left\{\phi_{\beta}, G\right\},
$$

Since, all constraints, including gauge one, have zero Dirac's brackets with everything, we can consider them as strong equations. This change of brackets reflects the reduction in number of degrees of freedom. It is easy verify that

$$
\sum_{i=1}^{n}\left\{q_{i}, p_{i}\right\}_{P . B .}=n \quad \sum_{i=1}^{n}\left\{q_{i}, p_{i}\right\}_{D}=n-m
$$

So the Dirac brackets take into account the constrained character of the theory and effectivelly reduce the phase space.

As it has been mentioned in the introduction, this method works only for gauges, known as unitary gauges [3]. To overcome this restriction one can proceed as follows. Suppose, the gauge condition depends on derivatives of the $r+l$ - th order of coordinates with respect to the time

$$
\chi_{\alpha}\left(p, q^{(0)}, q^{(1)}, q^{(2)}, \ldots, q^{(r+l)}\right)=0, \quad q^{(k)} \equiv \frac{d^{k}}{d t^{k}} q(t)
$$

The main idea is to consider the enlarged configuration space by rising these higher order derivatives the status of coordinates. This can be achieved by identifyning the initial theory with equivalent theory with higher derivatives and applying to it the Ostrogradsky method for construction of the phase space. More precisely, suppose that $r$ - is the maximum of the order of derivative with respect to the time $q^{(r)}$ entering in to the Lagrangian ${ }^{(r)} L\left(q, q^{(1)}, \ldots, q^{(r)}\right)$ while the highest order of time derivative in gauge fixing condition is $r+l$. Then, instead of do the Legendre transformation on the coordinates of the configuration space of the initial Lagrangian $L\left(q, \ldots, q^{(r)}\right)$ we suggest dealing with the Legendre transformation for the new Lagrangian ${ }^{(r+l)} L^{*}$ defined as a function of fictional auxiliary variables $q^{(r+1)}, \ldots, q^{(r+l 1)}$ by the anzatz

$$
{ }^{(r+l)} L^{*}\left(q, q^{(1)}, \ldots, q^{(r+l)}\right)={ }^{(r)} L\left(q, q^{(1)}, \ldots, q^{(r)}\right)
$$

The phase obtained for the new equivalent theory will be an extended phase space for the initial system. And our gauge fixing conditions (2.8), nonunitary in the ordinary phase space, now becomes the usual unitary gauge in the extended phase space. To prove this, let us briefly describe the Ostrogradsky method of the Hamiltonian formulation of theories with higher derivatives [3], [6]. 


\section{OSTROGRADSKY METHOD FOR THEORIES WITH HIGHER DERIVATIVES}

Let us consider the variational problem for the unconstrained mechanical system $t$

$$
S[q]=\int d t L,
$$

descrbed by the Lagrangian $L\left(q, q^{(1)}, q^{(2)}, \ldots, q^{(k)}\right)$ which is a function not only of coordinate $q$ and velocity $\dot{q}$ but also of higher derivatives of coordinate with respect to the time. The Euler - Lagrange equation follows from the extremum condition $\delta S=0$

$$
\sum_{s=0}^{k}(-1)^{s} \frac{d^{s}}{d t^{s}}\left(\frac{\partial L}{\partial q^{(s)}}\right)=0
$$

with the zero boundary conditions for variations $\left.\delta q^{(i)}\right|_{\text {boundary }}=0 i=0, \ldots, k-1$. Thus, in general the Euler - Lagrange equation has order $2 k$. The Ostrogradsky theorem stands that there is a generalized Legendre transformation such that this Euler - Lagrange equations for the non - singular $n$ - dimensional system (3.9) can be transformed into the equivalent set of first order equations of the Hamiltonian form on a space of dimension $2 \mathrm{kn}$. To construct the Legendre transformation, let us introduce $2 k n$ canonical variables $\xi_{i}$ and $p_{i} i=1, \ldots, k$

$$
\begin{aligned}
& \xi_{i}=q^{(i-1)} \\
& p_{i}=\sum_{s=i}^{k}(-1)^{s-i} \frac{d^{s-i}}{d t^{s-i}}\left(\frac{\partial L}{\partial q^{(s)}}\right)
\end{aligned}
$$

and then set the function

$$
H(\xi, \dot{\xi})=-L+\sum_{i=1}^{k-1} p_{i} \xi_{i+1}+p_{k} \dot{\xi}_{k}
$$

where the Lagrangian $L$ in terms of new variables has the form

$$
L\left(q, q^{(1)}, q^{(2)}, \ldots, q^{(k)}\right)=L\left(\xi_{1}, \xi_{2}, \ldots, \xi_{k}, \dot{\xi_{k}}\right)
$$

Now, taking into account that for nonsingular Lagrangian we can express from (3.10) the velocity $\dot{\xi}_{k}$ as a function of the remaining $r$ variables

$$
\dot{\xi}_{k}=f(\xi, p)
$$

one can, according to Ostrogradsky's theorem [3], [6], rewrite the Euler - Lagrange equations (3.9) in the Hamiltonian form

$$
\begin{aligned}
\dot{\xi}_{i} & =\left\{\xi_{i}, \bar{H}\right\}, \\
\dot{p}_{i} & =\left\{p_{i}, \bar{H}\right\},
\end{aligned}
$$

${ }^{1}$ We assume that $\mathrm{q}$ is the $n$ - dimensional vector 
with the Hamiltonian $\bar{H}(\xi, p)$ defined by

$$
\bar{H}(\xi, p)=\left.H\right|_{\dot{\xi_{k}=f(\xi, p)}},
$$

and the Poisson brackets in $(3.13)$ have the form

$$
\{F, G\}=\sum_{s=1}^{k}\left(\frac{\partial F}{\partial \xi_{s}} \frac{\partial G}{\partial p_{s}}-\frac{\partial F}{\partial p_{s}} \frac{\partial G}{\partial \xi_{s}}\right)
$$

\section{ENLARGED PHASE SPACE FOR COVARIANT GAUGES}

Now we are ready to return to the case of the singular theory with the first class constraints (2.1) and to consider the covariant gauges of the type (2.8)

$$
\chi_{\alpha}\left(p, q, q^{(1)}, q^{(2)}, \ldots, q^{(l+1)}\right)=0
$$

Let us correlate with the initial singular theory, describing by Lagrangian $L(q, \dot{q})$ the equivalent theory for which the new Lagrangian is considered as a function of additional derivatives of the coordinate with respect to the time variable up to the l-th order ( $l$ is a maximal order of a time derivative in the gauge condition (2.8))

$$
L^{*}\left(q, q^{(1)}, \ldots, q^{(l)}\right)=L(q, \dot{q})
$$

The method of construction of phase space picture for non singular Lagrangians described in previous section admit the generalization to the singular case in Dirac's sense [3]. Application of the above written procedure to new Lagrangian $L^{*}$ gives the following. According to the definition of canonical conjugate variables (3.10), for the theory (4.15) we obtain the usual part of canonical variables $q, p$

$$
\begin{aligned}
& \xi_{1}=q^{(0)}=q, \\
& p_{1}=\frac{\partial L}{\partial q^{1}}=p
\end{aligned}
$$

and additional one $\xi_{i}^{*}, p_{i}^{*}, \quad i=2, \ldots, l+1$

$$
\begin{aligned}
\xi_{i}^{*} & =q^{(i-1)} \\
p_{i}^{*} & =\sum_{s=i}^{l+1}(-1)^{s-i} \frac{d^{s-i}}{d t^{s-i}}\left(\frac{\partial L^{*}}{\partial q^{(s)}}\right),
\end{aligned}
$$

From (4.17) it follows that in addition to the old constraints (2.1) we obtain a new set of $l$ constraints

$$
p_{i}^{*}=0, \quad i=2, \ldots l+1
$$


Taking into account that the new canonical Hamiltonian coincides with the initial one

$$
H_{C}^{*}\left(\xi^{*} p^{*}\right)=H_{C}\left(\xi_{1}, p_{1}\right)
$$

one can verify that there aren't new secondary constraints, and we obtain the extended Hamiltonian for the new theory in terms of the old one $H_{E}\left(\xi_{1}, p_{1}\right)$

$$
H_{E}^{*}\left(\xi^{*} p^{\star}\right)=H_{E}\left(\xi_{1}, p_{1}\right)+\sum_{s=2}^{l+1} u_{s}^{*} p_{s}^{*},
$$

with new Lagrangian multipliers $u^{*}$. So in the extended phase space $\left(\xi^{*} p^{*}, \xi_{1}, p_{1}\right)$ we get the generalized dynamics with the set of constraints

$$
\begin{aligned}
\phi_{\alpha}(p, q) & =0, \quad \alpha=1, \ldots, m, \\
p_{i}^{*} & =0, \quad i=2, \ldots, l+1,
\end{aligned}
$$

with the desired condition (2.8), that has the form of a unitary gauge depending only on canonical variables

$$
\chi_{\alpha}\left(\xi^{*}, p^{*}, \xi_{1}, p_{1}\right)=0 .
$$

Now if we wants to reduce the phase space of our theory due to the appearance of new constraints (4.18), we need additional $l$ - gauge conditions

$$
\chi_{\alpha}^{\star}\left(\xi_{1}, p_{1}, p^{*}, \xi^{*}\right)=0, \quad \alpha=2, \ldots, l+1 .
$$

\section{Gauge $x_{\mu} \dot{x}^{\mu}=0$ FOR A Relativistic Particle}

In this section, we will apply the above described scheme to the simple example, the relativistic particle with mass $m$

$$
W[x]=-m \int_{0}^{T} d \tau \sqrt{\dot{x}_{\mu}^{2}} .
$$

This action is invariant under reparametrization of time

$$
\begin{aligned}
\tau \rightarrow \tau^{\prime} & =s(\tau), \\
x(\tau) \rightarrow x\left(\tau^{\prime}\right)^{\prime} & =x(\tau),
\end{aligned}
$$

with $d s / d \tau>0$. Therefore, there is an arbitrary function in the solution for the equation of motion. As a consequence of this invariance, we have identically vanishing canonical Hamiltonian and energy shell constraint

$$
\varphi^{1} \equiv p^{2}-m^{2}=0 .
$$


Thus, the total Hamiltonian is

$$
H_{T}=u(t)\left(p^{2}-m^{2}\right) .
$$

There is some freedom in the definition of dynamics in the reparametrized invariant theory. This fact is reflected in the gauge fixing procedure. It is known that in this case the gauge fixing condition with necessity explicitly depends on the time parameter. For example, the proper time fixing

$$
x_{0}(\tau)=\tau
$$

corresponds to the instant form of dynamics for relativistic particle. In this gauge, one can according to (2.6), fix the function $u(t)$

$$
u(t)=\frac{1}{2 \sqrt{p_{i}^{2}+m^{2}}}
$$

and get the usual equations of motion

$$
\dot{x}_{i}=\frac{p_{i}}{\sqrt{p_{i}^{2}+m^{2}}}
$$

governed by the reduced Hamiltonian $H_{R e d}=\sqrt{p_{i}^{2}+m^{2}}$ [ $]$. How cfn one can get this results in a gauge with the dependence onthe velocity $\dot{x}^{\mu}$, for example, of a following type:

$$
\xi \equiv \dot{x}^{\mu} x_{\mu}=0
$$

To do this, we will act in the spirit of the previous section. Let us pass from the initial singular theory, described by the action (5.22) to the equivalent theory for which the new Lagrangian is considered as a function of a first and second order derivatives of coordinates with respect to the time. After the transformation to the enlarged configuration space with the coordinates

$$
\begin{gathered}
\xi_{\mu}=x_{\mu}, \quad \xi_{\mu}^{*}=\dot{x}_{\mu}, \\
L^{*}\left(\xi, \xi^{*}, \dot{\xi}^{*}\right)=-m \int_{0}^{T} d \tau \sqrt{\xi_{\mu}^{* 2},}
\end{gathered}
$$

we see that in addition to the old constraints (5.24) there are new ones

$$
p_{\mu}^{*}=\frac{\partial L^{*}}{\partial \xi_{\mu}^{*}}=0
$$

Taking into account that new secondary constraints do not arise we obtain the extended Hamiltonian for the new theory

$$
H_{E}^{*}=u(t)\left(p^{2}-m^{2}\right)+u_{\nu}^{*} p_{\nu}^{*}
$$


with the new Lagrangian multipliers $u_{\nu}^{*}$. The gauge condition $\dot{x}^{\mu} x_{\mu}=0$ in the enlarged phase space has now the form of unitary gauge

$$
\chi=\xi_{\mu}^{*} \xi^{\mu}=0
$$

Let us choose additional gauge conditions for elimination of new constraints (5.29)

$$
\chi_{\nu}^{\star} \equiv \xi_{\nu}^{*}-a_{\nu}(\tau)=0
$$

with some functions $a_{\nu}(\tau)$. With the help of these gauge conditions one can find the Lagrange multipliers and verify that the choice of this vector leads to one or another form of dynamics. The choice $a_{\mu}(\tau)=(a(\tau), 0,0,0)$ corresponds to the instant form of dynamics with the time variable $x_{0}=a(\tau)$ while for the light - like vector $a_{\mu}(\tau)=(a(\tau), 0,0, a(\tau))$ we get the light cone formulation for the relativistic particle dynamics with the light cone time $x_{+} \equiv x_{0}+x_{3}=$ $2 a(t)$.

The authors would like to thank A.N. Kvinikhidze, V.V. Nesterenko and Yu.S. Surovtsev for helpful comments and discussions.

\section{REFERENCES}

[1] P.A.M. Dirac, Lectures on Quantum Mechanics. Belfer Graduate School of Science, (Yeshive University, New York, 1964).

[2] K. Sundermeyer Constrained Dynamics, Lecture Notes in Physics N 169, Springer Verlag, Berlin - Heidelberg - New York, 1982.

[3] D.M. Gitman, I.V. Tyutin, Quantization of Fields With Constraints Springer Verlag, Bonn, 1990.

[4] L.G. Yaffe, Lett. Nuovo. Cim. 18, (1977), 561

[5] E.S. Fradkin, G.A. Vilkovisky, CERN Preprint Ref.TH.2332 (1977).

[6] B.A. Dubrovin. S.P. Novikov, A.T. Fomenko, Modern Geometry - Methods and Aplications, Part II, The Geometry and Topology of Manifold Springer Verlag, New York, 1984.

[7] A.J. Hanson, T. Regge, C. Teitelboim Constrained Hamiltonian Systems, , Accademia Nazionale de Lincei, 1976 